DOI: $10.1002 /(($ please add manuscript number $))$

Article type: Communication

\title{
Self-assembly of an amphiphilic $\pi$-conjugated dyad into fibers: ultrafast and ultrasensitive humidity sensor.
}

Marco A. Squillaci, Laura Ferlauto, Yulian Zagranyarski, Silvia Milita, Klaus Müllen, and Paolo Samori*

M. A. Squillaci, L. Ferlauto, Prof. P. Samorì

ISIS \& icFRC, University of Strasbourg \& CNRS, 8 Allée Gaspard Monge, 67000,

Strasbourg, France.

E-mail: samori@unistra.fr

Y. Zagranyarski, Prof. K. Müllen

Max Planck Institute for Polymer Research, Ackermannweg 10, 55128, Mainz, Germany.

L. Ferlauto, Dr. S. Milita

Istituto per la Microelettronica e Microsistemi (IMM) - CNR, via Gobetti 101, 40129, Bologna, Italy.

Keywords: self-assembly, amphimphilicity, supramolecular chemistry, monomolecular dyad, humidity sensor

Humidity sensors are of paramount importance in numerous science and technology fields thus are commonly used in industries, hospitals, environmental monitoring, etc. ${ }^{[1]}$ Hitherto, different transduction mechanisms have been explored in order to improve sensitivity, response speed and recovery time, by exploiting different properties as read-out, including optical $^{[2]}$ and electrical characteristics (capacitance, ${ }^{[3]}$ resistance ${ }^{[4]}$, gate effect in field-effect transistors ${ }^{[5]}$ ) as well as small mass changes. ${ }^{[6]}$ To detect water in the environment, numerous materials have been employed as active components, including polymers, ${ }^{[7]}$ carbon based materials ${ }^{[8]}$ and composites. ${ }^{[9]}$ Since the sensing occurs via molecular recognition, the sensitivity in humidity sensing can, in principle, be harnessed by using low dimensional structures exhibiting a high surface area, fully decorated with receptors. 


\section{WILEY-VCH}

Amphiphilic molecular design is a viable strategy to achieve high degree of order at the supramolecular level, via sub-nm phase segregation between hydrophobic and hydrophilic moieties, to ultimately modulate the properties of organic electronic materials. ${ }^{[10]}$ Amphiphilic molecules with large $\pi$-conjugated cores have been employed to obtain excellent optoelectronic properties (e.g. charge transport, light-emission, etc.) associated with characteristic nanostructures. Typical examples include hexa-peri-hexabenzocoronene $(\mathrm{HBC}),{ }^{[11]}$ perylene diimide, ${ }^{[12]}$ porphyrin, ${ }^{[13]}$ and fullerene ${ }^{[14]}$ based amphiphiles, which tend to possess high charge carrier mobilities when are self-assembled into ordered nanostructures. Donor-acceptor dyads are well known molecular systems that can undergo self-assembly, forming fibers exhibiting good (photo)electrical characteristics. ${ }^{[15]}$ This self-assembly capability, render them interesting components for technological applications in (opto)electronics, including thin-film transistors, ${ }^{[16]}$ photovoltaics ${ }^{[17]}$ and light-emitting diodes. ${ }^{[18]}$

In this work we report the light-triggered self-assembly of an amphiphilic electron donoracceptor (D-A) dyad, based on a conjugated backbone containing an oligothiophene (OT) and a perylenediimide (PDI), connected by a conformationally rigid ethynylene spacer, forming fibers (Figure 1). These architectures, exposing ethylene glycol in their external shell, are employed as active components in humidity sensors that exhibit high sensitivity and ultrafast response.

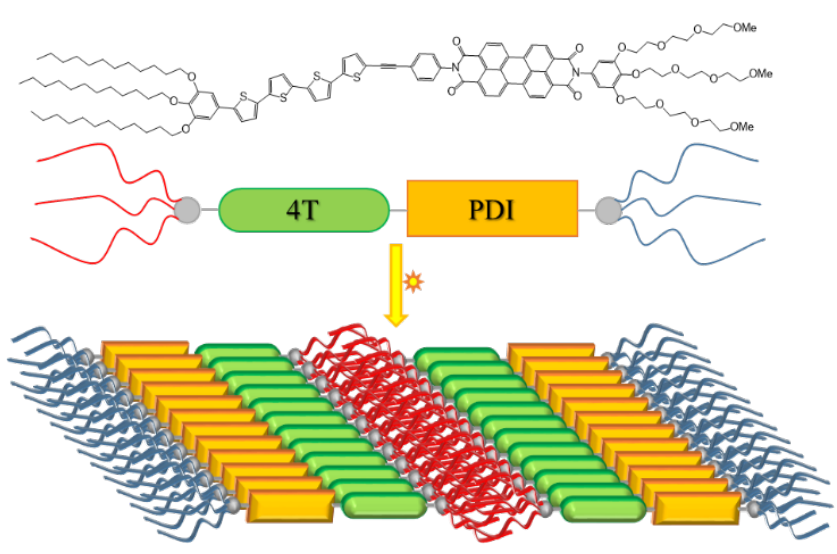




\section{WILEY-VCH}

Figure 1: Molecular structure and packing model of PDI-4T dyad.

The synthesis of the PDI-4T dyad was accomplished by a Sonogashira coupling of an ethynyl-group-terminated OT derivative with the corresponding iodophenyl-group-appended PDI derivatives, respectively, as described in literature ${ }^{[19]}$ MALDI-TOF mass spectra of PDI4T reveals single species with isotopic distributions in accordance with calculations (Figure S11-S12 in the SI) and also agrees well with data reported in literature. ${ }^{[1]}$

The PDI-4T self-assembles into fibers in solution, using a modified version of solventinduced precipitation (SIP), with a procedure different from the one reported in literature on the same system. ${ }^{[19]}$ In particular, $0.5 \mathrm{mg}$ of the dyad is dissolved in $1 \mathrm{ml}$ of THF. After complete dissolution, $200 \mu \mathrm{l}$ of the THF solution is added to $1 \mathrm{ml}$ of deionized water.

Differently from the procedure previously reported for generating fibers - with a length of just a few hundreds of nanometers - out of the PDI-4T dyad, ${ }^{[19]}$ our approach relies on a solution which is stable in environmental condition for several months. In particular, upon storage under dark condition, our solution retains its orange color (Figure S5) and does not exhibit traces of precipitate. When this solution is applied to the basal plane of a $\mathrm{SiO}_{2}$ substrate by drop-casting, amorphous films are obtained (Figure S1 in the supporting information).

By keeping the solution in a sealed glass vial on the lab bench, thus under daily light for at least one week a transition to light pink colour was observed. Such a colour transition of the solution can be accelerated upon exposure to the white light of a solar simulator (power density $=100 \mathrm{~mW} / \mathrm{cm}^{2}$ ) for at least 10 hours: the water solution slowly turns from orange to light pink (see Figure S5 and S6 in the SI), a behavior which may be ascribed to a modification on the molecular aggregation. 


\section{WILEY-VCH}

To gain an insight into such process on a smaller scale, we have performed a microscopic study as a function of the irradiation time. Atomic Force Microscopy (AFM) imaging of a drop cast film of the solution, after irradiation for a few minutes, revealed the formation of small nanoribbons with a length of few hundreds of nanometers which are randomly orientated on the $\mathrm{SiO}_{2}$ surface (Figure 2A, and Figure $\mathbf{S 2}$ in the SI).

Exposure to white light for about 10 hours promotes the aggregation of the ribbons into larger agglomerates (Figure 2B-D). In particular, from the SEM images in Figure 2C,D, it is possible to recognize the shape of the nanoribbons inside the aggregates (see also Figure $\mathbf{S 3}$ for a zoom-in).

Irradiation for 15 hours leads to the formation of longer bundles with a higher aspect ratio (Figure 2E), characterized by an average length of ca. $17.1 \mu \mathrm{m}$ and a width of ca. $1.97 \mu \mathrm{m}$. When the solutions are irradiated for over 20 hours, bundles of highly oriented ribbons are generated (see Figure 2F and Figure S4 in SI), appearing as macroscopic fiber networks featuring a dendritic shape with a length of several millimeters and an average width of ca. $1.65 \mu \mathrm{m}$. Overall, the series of images shown in Figure 2 provide for the first time a timeresolved evidence of the light-dependent evolution of the self-assembly: upon increase of the irradiation time of the solution, the aggregation of the nanoribbons into larger and larger fiber's bundles is promoted. 
WILEY-VCH
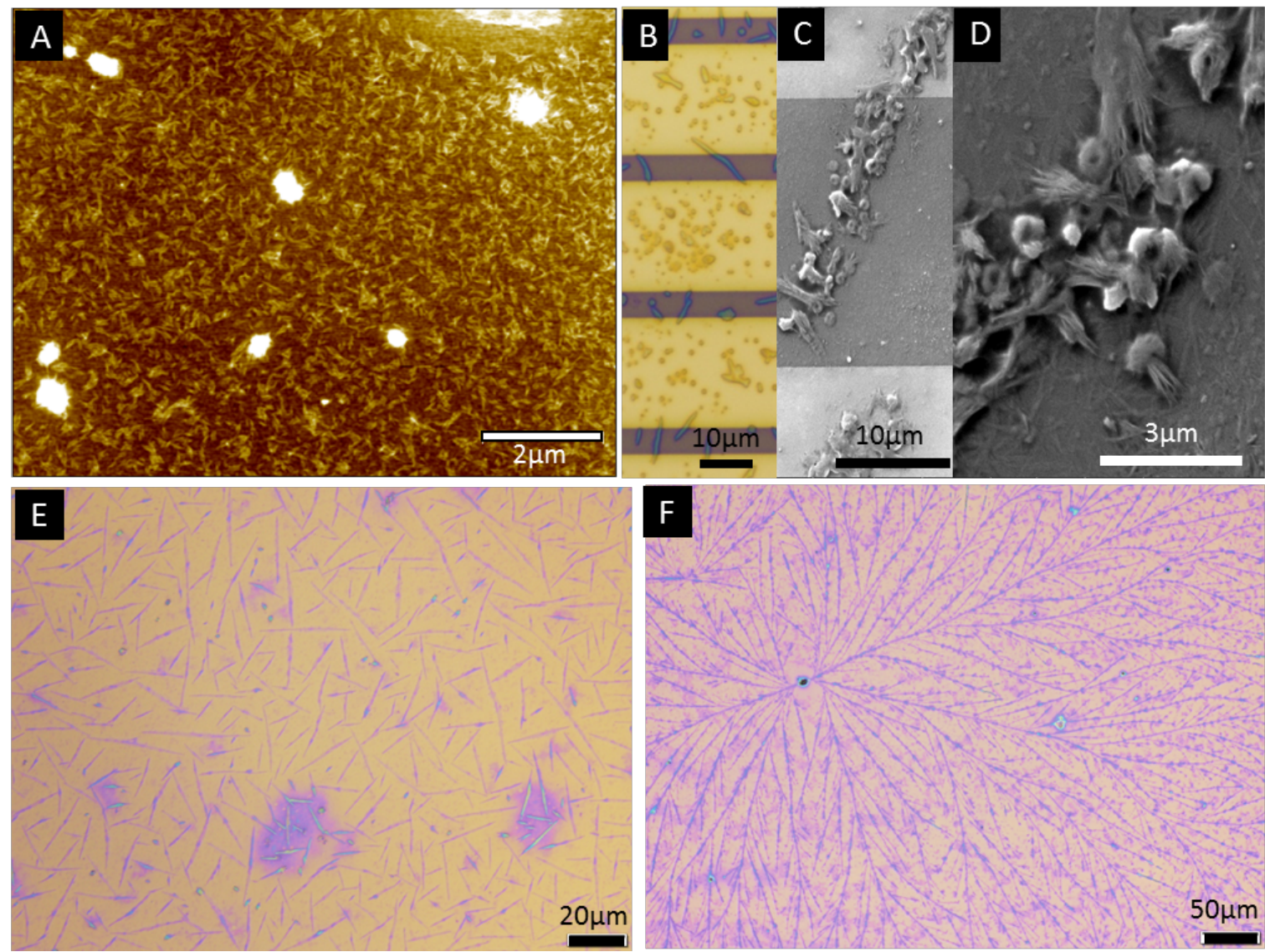

Figure 2: (A) AFM, (B,E,F) optical microscopy, and (C,D) SEM images of PDI-4T bundles of fibers at different aggregation levels obtained by exposure of the solution to the white light of a solar simulator for (A) few minutes, $(B, C, D) 10$ hours, (E) 15 hours, $(F)$ more than 20 hour).

Zoom-in with AFM in the less coated part of the films reported in Figure 2B-D, revealed the presence of laterally packed and intercoiled ribbons (Figure 3A) with lengths of several micrometers. From the cross section profiles in Figure 3B it is possible to identify 2 different populations of ribbon widths amounting to ca. $7.0 \mathrm{~nm}$ and $14.5 \mathrm{~nm}$. Being the contour length of the molecules when the side chains adopt a fully elongated conformation around $8 \mathrm{~nm},{ }^{[19]}$ our result can be interpreted by an interdigitation of the alkyl substituents belonging to adjacent ribbons and eventual coiling of the side-groups. The $14.5 \mathrm{~nm}$ width suggests a supramolecular organization characterized by two laterally assembled molecules, in which all 


\section{WILEY-VCH}

the hydrophobic side chains are in the middle of the structure, shielded from the water, as shown in Figure 1.
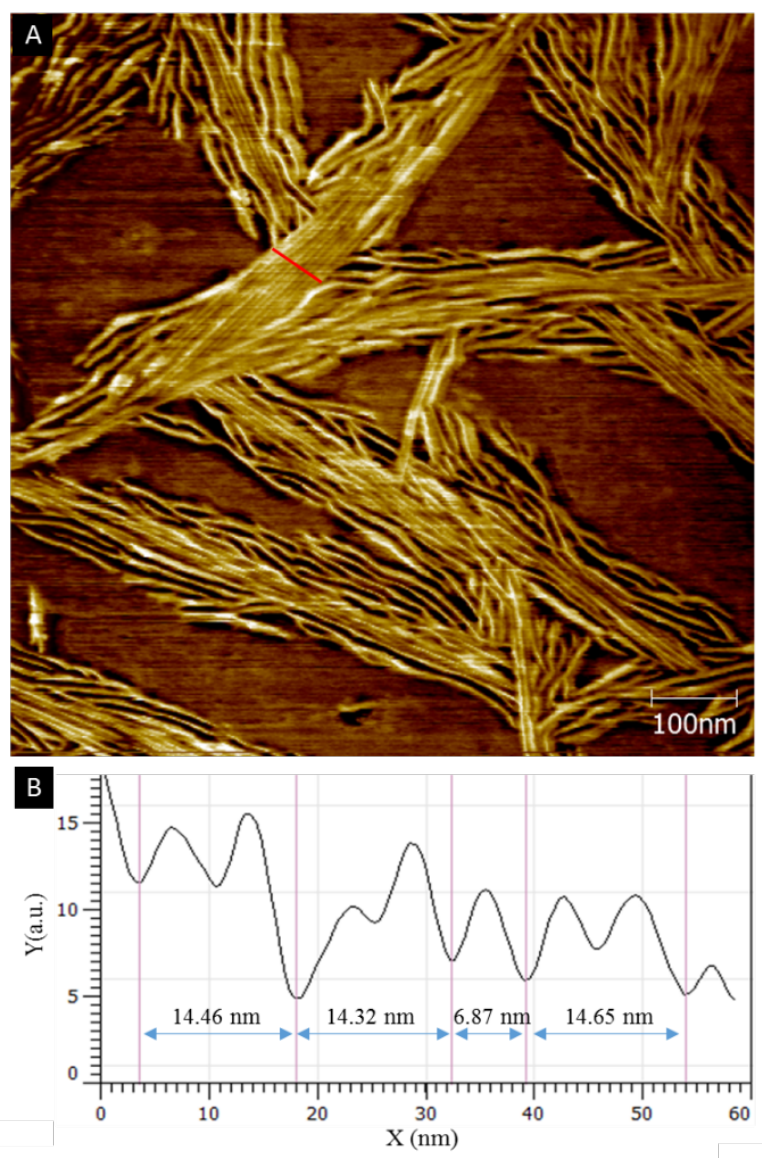

Figure 3: (A) AFM phase image of PDI-4T nanoribbons on $\mathrm{SiO}_{2}$ substrate. (B) Cross-section profile of the bundles (along the red line).

Interestingly, the use of water based SIP turned out to be key to the fiber formation: irradiation of PDI-4T solutions in either THF, $\mathrm{CHCl}_{3}$, DMF, Acetone, Ethanol or Isopropanol has not led to formation of fiber-like structures, even after over 50 hours of irradiation. It is also noteworthy that the light irradiation, together with the effect of the water environment, on an amphiphilic molecule, promotes the self-assembly into a fibrillar system without need of using chlorinated solvents that, upon exposition to white light, form radicals, which could be transferred to the molecules, promoting the self-assembly. ${ }^{[20]}$ In our case, the key role 


\section{WILEY-VCH}

played by light irradiation, in the fibers growth, likely involves electron transfer and electrostatic interactions. However, X-Ray Photoelectron Spectroscopy (XPS) analysis, on PDI-4T drop-cast solutions before and after irradiation, provided evidence for an increase of the peak at $168.2 \mathrm{eV}$ (Figure S10 in SI), being unambiguously assigned to a fully oxidized sulfur, up to $46.4 \%$, demonstrating that an intense light exposure leads also to an oxidization of the thiophene rings in the molecule. To get a deeper insight into the order at the supramolecular level within the fiber's bundles, we have carried out Grazing incidence XRay Diffraction (GIXRD) investigations of the films shown in Figure 2F. The 2D X-ray pattern in Figure 4, presents a main peak at position $\mathrm{q}_{\mathrm{xy}}=1.78 \AA^{-1}$ which corresponds to a dspacing of $3.5 \AA$, typical distance for $\pi$ - $\pi$ stacking. Since the peak is perturbed by the large footprint of the X-ray beam, a realistic estimation of the lateral dimension of the crystalline domains is not possible. ${ }^{[21]}$ Being in the in-plane direction, this peak indicates that the dyad molecules adopt an edge-on orientation with respect to the basal plane of the substrate, in agreement with the proposed model in Figure 1. 


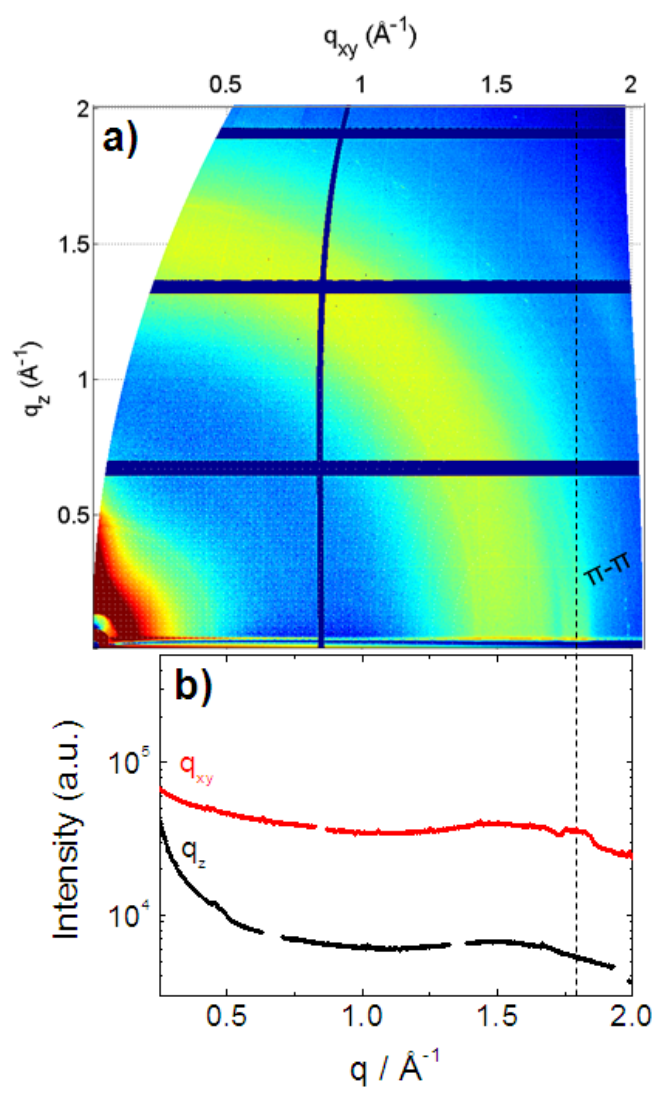

Figure 4: (a) 2D-GIXRD pattern of PDI-4T bundles of fiber together with (b) corresponding scattering intensity integrated along the out-of-plane $\left(q_{z}\right)$ and in-plane $\left(q_{x y}\right)$ directions.

To probe if the external shell of the fiber bundles consists of either hydrophobic alkyl based or more hydrophilic ethylene glycol based units, we have exposed these architectures to a humid environment. Such an exposure revealed a high sensitivity to the presence of water molecules in the atmosphere, confirming that the hydrophilic chains are in the external part of the ribbons, ${ }^{[11]}$ enabling dipole-dipole interactions and, in particular, hydrogen bonding, with water molecules. These interactions are likely strong enough to compress the molecules together inside the aggregate, decreasing the distance of the $\pi$ - $\pi$ stacking, in accordance with a previous XRD study. ${ }^{[1 \mathrm{~b}]}$ The decreasing $\pi-\pi$ distance leads to an exponential increase of the charge transport through the ribbons, thus of the conductivity of the self-assembled architectures. Therefore, the conductivity can be tuned with the relative humidity of the 


\section{WILEY-VCH}

environment: thus our system can be employed as resistive-type organic humidity sensor.

Noteworthy, the adsorption and desorption of water on the fibers surface is a fully reversible process.

The response rate, the sensitivity and the range of sensed humidity levels, were measured by depositing the fiber's bundles by a drop casting onto a $\mathrm{SiO}_{2} / \mathrm{Si} \mathrm{n}^{++}$substrates $(230 \pm 10) \mathrm{nm}$ thick $\mathrm{SiO}_{2}$, Fraunhofer Institute for Photonic Microsystems IPMS, Dresden, Germany), which were freshly cleaned and ozone-treated before being used. The dielectric surface exhibits patterned interdigitated gold electrodes (channel lengths $\mathrm{L}=2.5$ to $20 \mu \mathrm{m}$ ). The response speed was measured by sending a pulsed flow of humid air over the sample during the application of a constant bias of $5 \mathrm{~V}$. The results portrayed in Figure 5A show a response rate of about $26 \mathrm{~ms}$ with a good recovery time and an ultimate $100 \%$ reversibility. The calibration of the devices was carried out inside a chamber with a controlled atmosphere, in which the relative humidity $(\mathrm{RH})$ can be increased from 0 to $75 \%$ while performing the electrical measurement in real time. Figure 5B displays the results of the calibration for two different channel lengths using the same applied voltage. These measurements demonstrate that, when larger channel lengths are used, the sensitivity for higher humidity levels increases, as a result of the greater surface area of the fibers covered with hydrophilic ethylene glycol groups that can interact with the water molecules.

The calibration plot in Figure 5B allowed us to quantify the dependence of the measured current upon the humidity in the chamber. It reveals an exponential growth. The measured values of current goes from a few pA at $0 \% \mathrm{RH}$ up to tens of $\mathrm{mA}$ at $75 \%$, with an overall current variation exceeding 7 orders of magnitude. 

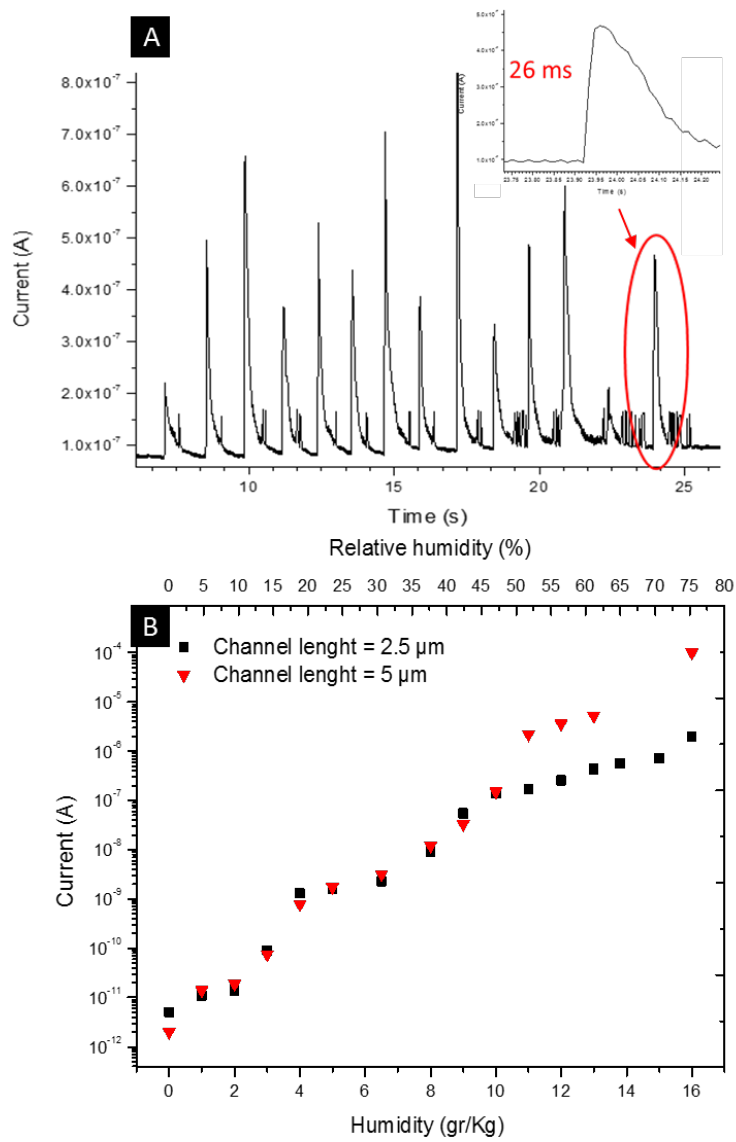

Figure 5: Characterization of PDI-4T humidity sensors. (A) Response speed under a pulsed flux of humid air during the application of a constant bias of $5 \mathrm{~V}$ (channel length $=5 \mu \mathrm{m})$. (B) Calibration of the response current in different environment (applied bias $=5 \mathrm{~V}$, temperature $=26.6^{\circ} \mathrm{C}$ )

Noteworthy is also that the current response is strongly selective for water molecules that, being donor of two hydrogen bonding, can behave as linker between two PEG side-chains exposed on two adjacent molecules within the stack. The plots reported in Figure S9 show the electrical response of our PDI-4T based humidity sensors when exposed to vapor of different solvents such as ethanol, methanol and ethyl ether. Among them, only methanol, being the smallest molecule and being able to act as hydrogen bonding donor, exhibited an increased current passing through the fibers. However, since methanol can donate just one 


\section{WILEY-VCH}

proton to create a hydrogen bonding, the change in the current is negligible compared to the one given by water under the same condition.

In summary, we have devised a novel approach, based on light irradiation acting as a remote control on the self-assembly of an amphiphilic D-A $\pi$-conjugated dyad into ordered structures. In particular, the joint effect of light irradiation and solvent induced precipitation in an environmentally friendly water solvent, makes it possible to form nanoribbons. Upon tuning the irradiation time, the size of aggregation of the ribbons, forming fiber bundles, can be modulated. These ultra-long 1D architectures possess an external shell exposing hydrophilic ethylene glycol moieties and $\pi-\pi$ stacking distances that decrease with the increasing humidity. ${ }^{[1 b]}$ Such architectures, when deposited on solid surfaces, reveal a high selectivity to water in the environment: the resistive sensor exhibits ultrafast response and extremely high sensitivity. To the best of our knowledge, the 7 order of magnitude change in current signal by varying the $\mathrm{RH}$ from $0 \%$ to $75 \%$ is the highest reported in literature for resistive humidity sensors based on organic molecules. Moreover, the response speed of 26 $\mathrm{ms}$ is in line with the best data recently reported on capacitive sensors based on graphene oxide continuous films exhibiting $30 \mathrm{~ms}$ response speed ${ }^{[8]}$ and on resistive sensors integrating organic fibers featuring a $10 \mathrm{~ms}$ response speed, being the fastest ever reported. ${ }^{[1 b]} \mathrm{Current}$ effort in our laboratories is on the integration of these architectures in flexible sensing devices.

Experimental Section: see Supporting Information

\section{Supporting Information}

Includes methods and materials, AFM images of film of PDI-4T after different exposure, macroscopic evidence of the aggregation, optical characterization, device fabrication and characterization, XPS and MALDI-TOF spectra. Supporting Information is available from the Wiley Online Library or from the author. 


\section{Acknowledgements}

We thank Dr. Long Chen, Dr. Ruomeng Duan and Christoph Sonn for their contribution in the early synthetic attempts and Dr. Fabiola Liscio for enlightening discussions on the XRD part. This work was financially supported by the EC through the European Re-search Council project SUPRAFUNCTION (GA-257305), the Marie-Curie ITN projects GENIUS (PITNGA-2010-264694) and iSwitch (GA no. 642196) the Agence Nationale de la Recherche through the LabEx project Chemistry of Complex Systems (ANR-10-LABX-0026_CSC), and the International Center for Frontier Research in Chemistry (icFRC).

Received: ((will be filled in by the editorial staff))

Revised: ((will be filled in by the editorial staff))

Published online: ((will be filled in by the editorial staff))

[1] a) J. Chu, X. Peng, P. Feng, Y. Sheng, J. Zhang, Sens. Actuators, B 2013, 178, 508; b) U. Mogera, A. A. Sagade, S. J. George, G. U. Kulkarni, Sci Rep 2014, 4, 4103; c) E. Traversa, Sens. Actuators, B 1995, 23, 135; d) Y. H. Wang, R. W. Besant, C. J. Simonson, W. Shang, Sens. Actuators B 2006, 115, 93.

[2] W. C. Wong, C. C. Chan, L. H. Chen, T. Li, K. X. Lee, K. C. Leong, Sens. Actuators, $B$ 2012, 174, 563 .

[3] H. P. Hong, K. H. Jung, J. H. Kim, K. H. Kwon, C. J. Lee, K. N. Yun, N. K. Min, Nanotechnology 2013, 24, 085501.

[4] Q.-Y. Tang, Y. C. Chan, K. Zhang, Sens. Actuators, B 2011, 152, 99.

[5] F. Liang, L.-B. Luo, C.-K. Tsang, L. Zheng, H. Cheng, Y. Y. Li, Mater Res Bull 2012, 47, 54.

[6] J. Xie, H. Wang, Y. Lin, Y. Zhou, Y. Wu, Sens. Actuators, B 2013, 177, 1083.

[7] M. V. Kulkarni, S. K. Apte, S. D. Naik, J. D. Ambekar, B. B. Kale, Sens. Actuators, B 2013, 178, 140.

[8] S. Borini, R. White, D. Wei, M. Astley, S. Haque, E. Spigone, N. Harris, J. Kivioja, T. Ryhanen, ACS Nano 2013, 7, 11166.

[9] Y. Zilberman, R. Ionescu, X. Feng, K. Müllen, H. Haick, ACS Nano 2011, 5, 6743.

[10] a) Y. Yamamoto, T. Fukushima, Y. Suna, N. Ishii, A. Saeki, S. Seki, S. Tagawa, M. Taniguchi, T. Kawai, T. Aida, Science 2006, 314, 1761; b) W. Zhang, W. Jin, T. Fukushima, A. Saeki, S. Seki, T. Aida, Science 2011, 334, 340.

[11] J. P. Hill, W. Jin, A. Kosaka, T. Fukushima, H. Ichihara, T. Shimomura, K. Ito, T. Hashizume, N. Ishii, T. Aida, Science 2004, 304, 1481.

[12] a) Y. Che, A. Datar, K. Balakrishnan, L. Zang, J. Am. Chem. Soc. 2007, 129, 7234; b) X. Zhang, Z. Chen, F. Wu, J. Am. Chem. Soc. 2007, 129, 4886; c) X. Zhang, S. Rehm, M. M. Safont-Sempere, F. Würthner, Nat Chem 2009, 1, 623.

[13] a) T. Sakurai, K. Tashiro, Y. Honsho, A. Saeki, S. Seki, A. Osuka, A. Muranaka, M. Uchiyama, J. Kim, S. Ha, K. Kato, M. Takata, T. Aida, J. Am. Chem. Soc. 2011, 133, 6537 ; b) T. Sakurai, K. Shi, H. Sato, K. Tashiro, A. Osuka, A. Saeki, S. Seki, S. Tagawa, S. Sasaki, H. Masunaga, K. Osaka, M. Takata, T. Aida, J. Am. Chem. Soc. 2008, 130, 13812.

[14] A. Munoz, B. M. Illescas, M. Sanchez-Navarro, J. Rojo, N. Martin, J. Am. Chem. Soc. 2011, 133, 16758.

[15] a) Y. Hizume, K. Tashiro, R. Charvet, Y. Yamamoto, A. Saeki, S. Seki, T. Aida, J. Am. Chem. Soc. 2010, 132, 6628; b) J. Luo, J. Kim, J. Huang, Accounts Chem Res 


\section{WILEY-VCH}

2013, 46, 2225; c) T. Nishizawa, K. Tajima, K. Hashimoto, J. Mater. Chem. 2007, 17, 2440; d) H. L. Huang, C. E. Chou, Y. K. Che, L. G. Li, C. Wang, X. M. Yang, Z. H. Peng, L. Zang, J. Am. Chem. Soc. 2013, 135, 16490.

[16] R. Pfattner, E. Pavlica, M. Jaggi, S.-X. Liu, S. Decurtins, G. Bratina, J. Veciana, M. Mas-Torrent, C. Rovira, J. Mater. Chem. C 2013, 1, 3985.

[17] a) G. Chidichimo, L. Filippelli, Int J Photoenergy 2010, 2010, 1; b) T. Ye, R. Singh, H. J. Butt, G. Floudas, P. E. Keivanidis, ACS Appl Mater Interfaces 2013, 5, 11844.

[18] a) J. L. Segura, H. Herrera, P. Bäuerle, J. Mater. Chem. 2012, 22, 8717; b) F. Fuchs, M. Linares, C. de Vet, P. Leclere, R. Demadrille, B. Grevin, Adv Mater 2014, 26, 6416.

[19] W. S. Li, A. Saeki, Y. Yamamoto, T. Fukushima, S. Seki, N. Ishii, K. Kato, M. Takata, T. Aida, Chem Asian J 2010, 5, 1566.

[20] E. Moulin, F. Niess, M. Maaloum, E. Buhler, I. Nyrkova, N. Giuseppone, Angew Chem Int Ed Engl 2010, 49, 6974.

[21] D. M. Smilgies, J Appl Crystallogr 2009, 42, 1030. 


\section{WILEY-VCH}

The self-assembly of an amphiphilic monomolecular electron acceptor-donor dyad into electroactive $\pi-\pi$ stacked fibrillar structures can be triggered by irradiation with visible light. These fibers, exposing a hydrophilic ethylene glycol in their external shell, show unique characteristics as resistive humidity sensors that exhibit high sensitivity and ultrafast response

\section{Keyword}

Marco A. Squillaci, Laura Ferlauto, Yulian Zagranyarski, Silvia Milita, Klaus Müllen, and Paolo Samori*

Self-assembly of an amphiphilic $\pi$-conjugated dyad into fibers: ultrafast and ultrasensitive humidity sensor.

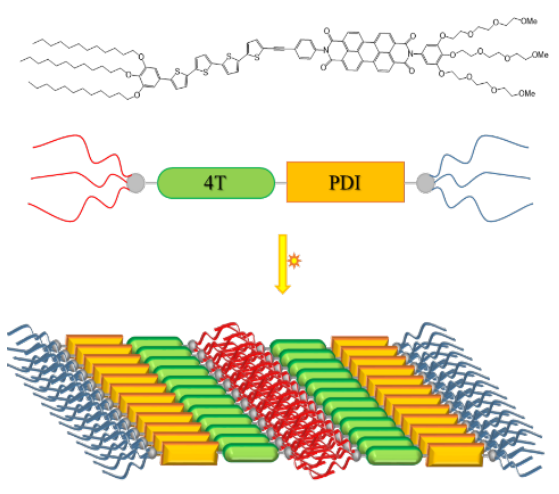

